\title{
Fluticasone furoate induced iatrogenic Cushing syndrome in a pediatric patient receiving anti-retroviral therapy
}

\author{
S A A van den Berg',2, N E van 't Veer', J M A Emmen' and R H T van Beek ${ }^{4}$ \\ 'Laboratory for Clinical Chemistry and Haematology, Amphia Hospital, Breda, The Netherlands, 2Laboratory \\ for Clinical Chemistry, Erasmus MC, Rotterdam, The Netherlands, Departments of ${ }^{3}$ Clinical Pharmacy, and \\ 4Pediatrics, Amphia Hospital, Breda, The Netherlands
}

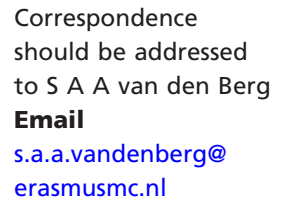

\section{Summary}

We present a case of iatrogenic Cushing's syndrome, induced by treatment with fluticasone furoate (1-2 dd, $27.5 \mu \mathrm{g}$ in each nostril) in a pediatric patient treated for congenital HIV. The pediatric patient described in this case report is a young girl of African descent, treated for congenital HIV with a combination therapy of Lopinavir/Ritonavir ( $1 \mathrm{dd} 320 / 80 \mathrm{mg}$ ), Lamivudine (1 dd $160 \mathrm{mg}$ ) and Abacavir (1 dd $320 \mathrm{mg}$ ). Our pediatric patient presented with typical Cushingoid features (i.e. striae of the upper legs, full moon face, increased body and facial hair) within weeks after starting fluticasone furoate therapy, which was exacerbated after increasing the dose to $2 \mathrm{dd}$ because of complaints of unresolved rhinitis. Biochemical analysis fitted iatrogenic Cushing's syndrome, with a repeatedly low cortisol $(<0.03 \mu \mathrm{M}$, ref $0.14-0.60 \mu \mathrm{M})$ and low ACTH ( $9 \mathrm{pg} / \mathrm{mL}$, ref $9-52 \mathrm{pg} / \mathrm{mL}$ ) without signs of adrenal insufficiency. No other biochemical abnormalities that could point to adrenal or pituitary dysfunction were detected; electrolytes, thyroid and gonadal function, and IGF-1 were within the normal range. Pharmacogenetic analysis revealed that the pediatric patient carried the CYP3A4 $* 1 B / * 1 G$ and CYP3A5 $* 3 / * 3$ genotype (associated with a partial and complete loss of enzyme activity, respectively) which is associated with the development of iatrogenic Cushing's syndrome in patients treated for HIV due to the strong inhibition of CYP3 enzymes by Ritonavir. Upon discontinuation of fluticasone treatment, the pediatric patient improved both clinically and biochemically with normalisation of cortisol and ACTH within a couple of weeks.

\section{Learning points:}

- Fluticasone therapy may induce iatrogenic Cushing's syndrome in a patient treated with anti-retroviral therapy.

- Pharmacogenetic analysis, in particular CYP3A genotyping, provides useful information in patients treated for HIV with respect to possible future steroid treatment.

- Fluticasone furoate is not detected in the Siemens Immulite cortisol binding assay.

\section{Background}

Intranasal corticosteroids are the preferred long-term therapy for allergic rhinitis/asthma in children. It suppresses inflammatory processes. Fluticasone furoate treatment is described to have no negative impact on the hypothalamic-pituitary-adrenal (HPA) axis as the systemic bioavailability is low (1). However, all clinical trials regarding adrenal function in patients using fluticasone furoate have been performed in patients using a 1 dose/day regimen (2), whereas a 2 dose/day is still within the recommended dose.

Also, it is generally overseen that a combination therapy of HIV anti-retroviral therapy and fluticasone 
propionate treatment may result in iatrogenic Cushing's syndrome, as some components of anti-retroviral therapy inhibit CYP3A4/5 enzyme activity (3).

\section{Case presentation}

The pediatric patient described here is a 6-year-old girl, treated for congenital HIV infection. She presented to the outpatient clinic with suspicion of nephrotic syndrome. She had a full moon face, increased hirsutism (especially in the face), nightly sweating and an increased appetite. Also her behaviour had changed over the previous weeks. No oedema was present. At first the parents declared that there had been no change in medication. At a follow-up appointment however, they confirmed that the pediatric patient had suffered from an obstructed nose, for which fluticasone furoate therapy was started once daily two months earlier, which had recently been increased to twice daily.

\section{Investigation}

Cortisol and ACTH concentrations were determined on several occasions in the follow-up of this case.

Upon presentation, cortisol and ACTH concentrations were low (random cortisol $<0.03 \mu \mathrm{M}$ (normal 0.14-0.60 $\mu \mathrm{M}$ ) and random ACTH 9 pM (normal 9-52 pM) but the pediatric patient did not show signs of adrenal insufficiency. Similar data were found one week later (random cortisol $<0.03 \mu \mathrm{M}$ and random ACTH 8 pM). To exclude adrenal insufficiency, early morning cortisol and ACTH concentrations were determined and both were found to be low (cortisol $<0.03 \mu \mathrm{M}$ and random ACTH $10 \mathrm{pM}$ ).

Given the use of an intranasal steroid, we determined that the biochemical phenotype best fitted with an iatrogenic Cushing syndrome, even though this was described not to be associated with fluticasone furoate use (2). Unfortunately, serum concentration of fluticasone furoate analysis was not available at the time of diagnosis. Pediatric patient was instructed to reduce the dosage from 2 dd to $1 \mathrm{dd}$ for 1 week and to discontinue use after that week.

In our opinion, an ACTH stimulation test would have been useful if the pediatric patient would have had signs of primary adrenal failure. Primary adrenal failure is commonly associated with low cortisol and high ACTH concentrations. In our case, ACTH levels were low.

Upon return visit, 2 weeks after discontinuation of therapy, morning cortisol concentration was still low $(<0.03 \mu \mathrm{M})$ but ACTH concentration was in the lownormal range $(15 \mathrm{pM}) .6$ weeks after discontinuation, morning cortisol was normalised $(0.32 \mu \mathrm{M})$ with a concordant ACTH concentration (15 pM).

Iatrogenic Cushing as well as adrenal suppression is uncommon upon fluticasone furoate therapy but had been described in patients treated with antiretroviral therapy due to competition for the rate limiting metabolizing enzyme CYP3A (4), we hypothesized that our pediatric patient may have a low level of expression of the gene.

Pharmacogenetic analysis by PCR-Restriction Fragment Length Polymorphism, for the * $1 \mathrm{~B},{ }^{*} 1 \mathrm{G},{ }^{*} 2,{ }^{*} 3$, * $4, * 5, * 6, * 10, * 12, * 17, * 18,{ }^{*} 20,{ }^{*} 22$ alleles of CYP3A4 and *3 allele of CYP3A5, revealed that the pediatric patient carried the $C Y P 3 A 4{ }^{*} 1 B /{ }^{*} 1 G$ phenotype, and was a CYP3A5 non-expressor.

\section{Treatment}

Upon presentation, pediatric patient was treated with fluticasone furoate (Avamys) for complaints of rhinitis and a combination therapy of Lopinavir/Ritonavir (1 dd 320/80 mg), Lamivudine (1 dd $160 \mathrm{mg}$ ) and Abacavir (1 dd $320 \mathrm{mg}$ ) for congenital HIV infection.

During the course of the case, fluticasone furoate treatment was reduced and stopped because of the development of Cushingoid features. A short tapering program (1 week) was chosen because of the rapid onset of disease and the lack of evidence for tapering in asthma and COPD patients (5).

\section{Outcome and follow-up}

Within a period of three months after discontinuation, the behaviour and clinical signs of Cushing normalised. Also her stamina improved and she could attend full day school.

No post-cessation treatment was initiated, but the pediatric patient was regarded as adrenal insufficient for a period of 3 months. Parents were instructed to contact the paediatric department in case the pediatric patient developed a high fever $\left(>39^{\circ} \mathrm{C}\right)$ or in event of trauma.

\section{Discussion}

Here, we present a case of iatrogenic Cushing's syndrome caused by fluticasone furoate treatment in a pediatric patient treated for congenital HIV.

Fluticasone furoate is an intranasal steroid spray for the treatment of allergic rhinitis (2). The furoate ester renders it poorly absorbable in the gastrointestinal 
tract and causes the minimal amount that is absorbed to be rapidly metabolized by the liver (1). Given these properties, adrenal suppression should be expected only when the bioavailable dose is such that circulating levels become high enough to interfere with the hypothalamicpituitary axis. Indeed, clinical trials have shown that adrenal suppression was absent in children treated with fluticasone furoate, although it must be said that all trials in which adrenal function in children was addressed were based on the lowest recommended dose (2).

Given the rapid onset and worsening of Cushingoid features in a young child, it was logical to discontinue steroid treatment as fast as possible.

To avoid steroid withdrawal symptoms, doctors often employ a tapering program when weaning patients from steroids to prevent adrenal suppression. Unfortunately, the duration of steroid therapy does not predict whether an individual pediatric patient will develop adrenal suppression upon discontinuation of treatment and gives no direction for tapering strategies (6). For example, at high doses, steroids may induce adrenal suppression within days. Also, it is often overlooked that inhaled steroid therapy may also result in adrenal suppression (7). Interestingly, in spite of the common practice of tapering, a large meta-analysis of different tapering regimens did not show benefit of tapering when compared to abrupt discontinuation in asthma or COPD patients (5).

For our pediatric patient, medication was tapered by reducing the dose for 1 week, after which treatment was stopped completely. Our pediatric patient responded with the typical biochemical response seen after steroid withdrawal (8); ACTH and cortisol concentration remained low during the first weeks after cessation and recovered thereafter. Fortunately, our pediatric patient did not experience any clinical withdrawal symptoms and within a period of 2 months, the behaviour and clinical signs of Cushing were all normalised.

We hypothesized that the rapid development of Cushingoid features, even at low administered steroid doses, was the result of slow metabolism of the fluticasone furoate. This most probably increased the circulating concentration, which was exacerbated when the daily dosage was increased from $1 \mathrm{dd}$ to $2 \mathrm{dd}$. The slow metabolism of the compound was most probably due to the pharmacogenetic profile of our pediatric patient, expressing the CYP3A4 ${ }^{*} 1 B /{ }^{*} 1 G$ and CYP3A5 ${ }^{*} 3 /{ }^{*} 3$ genotype. Although the relative contribution of CYP3A5 in drug metabolism remains a debate, it is beyond doubt that both Cyp3A4 and CYP3A 5 are involved in metabolism of fluticasone (9), and that the $C Y P 3 A 4{ }^{*} 1 B /{ }^{*} 1 G$ genotype results in low enzyme activity (10). Furthermore, similar to our case study, other case studies have been presented in the literature (4), and it has indeed been shown that ritonavir is a potent inhibitor of both CYP3A4 and CYP3A5 (3).

Alternative therapeutic options may be limited; a minimal local dose of intranasal steroids is needed to achieve a desirable effect. Antihistaminic therapy is contraindicated for the same reason as high-dose steroids due to their metabolism via CYP3A4. A treatment strategy with a low-dose antihistaminic agent in combination with low-dose steroids may be an option, but should be tailored to each individual pediatric patient.

\section{Declaration of interest}

The authors declare that there is no conflict of interest that could be perceived as prejudicing the impartiality of the research reported.

\section{Funding}

This research did not receive any specific grant from any funding agency in the public, commercial or not-for-profit sector.

\section{Patient consent}

A written informed consent statement was obtained for publication of this article.

\section{Author contribution statement}

van den Berg wrote the draft manuscript and performed laboratory analysis. van 't Veer consulted on the interaction of medical therapy. Emmen supervised and authorized laboratory analysis. van Beek is the physician who is responsible for the pediatric patient.

\section{References}

1 Derendorf H \& Meltzer EO 2008 Molecular and clinical pharmacology of intranasal corticosteroids: Clinical and therapeutic implications. Journal of Allergy and Clinical Immunology 63 1292-1300. (doi:10.1111/ j.1398-9995.2008.01750.x)

2 Tripathy I, Levy A, Ratner P, Clements D, Wu W \& Philpot E 2009 HPA axis safety of fluticasone furoate nasal spray once daily in children with perennial allergic rhinitis. Pediatric Allergy and Immunology 20 287-294. (doi:10.1111/j.1399-3038.2008.00775.x)

3 Granfors MT, Wang JS, Kajosaari LI, Laitila J, Neuvonen PJ \& Backman JT 2006 Differential inhibition of cytochrome P450 3A4, 3A5 and $3 \mathrm{~A} 7$ by five human immunodeficiency virus (HIV) protease inhibitors in vitro. Basic and Clinical Pharmacology and Toxicology 98 79-85. (doi:10.1111/j.1742-7843.2006.pto_249.x)

4 Mahlab-Guri K, Asher I, Gradstein S, Zung A, Radian-Sade S, Elbirt D \& Sthoeger Z 2011 Inhaled fluticasone causes iatrogenic Cushing's syndrome in patients treated with Ritonavir. Journal of Asthma $\mathbf{4 8}$ 860-863. (doi:10.3109/02770903.2011.606580)

5 Richter B, Neises G \& Clar C 2002 Glucocorticoid withdrawal schemes in chronic medical disorders. A systematic review. Endocrinology and Metabolism Clinics of North America 31 751-778. (doi:10.1016/S0889. 8529(02)00008-7) 
6 Livanou T, Ferriman D \& James VHT 1967 Recovery of hypothalamopituitary-adrenal function after corticosteroid therapy. Lancet 290 856-859. (doi:10.1016/S0140-6736(67)92592-5)

7 Molimard M, Girodet P-O, Pollet C, Fourrier-Réglat A, Daveluy A, Haramburu F, Fayon M \& Tabarin A 2008 Inhaled corticosteroids and adrenal insufficiency: prevalence and clinical presentation. Drug Safety 31 769-774. (doi:10.2165/00002018-20083109000005)

8 Graber AL, Ney RL, Nicholson WE, Island DP \& Grant WL 1965 Natural history of pituitary-adrenal recovery following long-term suppression with corticosteroids. Journal of Clinical Endocrinology and Metabolism 25 11-16. (doi:10.1210/jcem-25-1-11)

9 Pearce RE, Leeder JS \& Kearns GL 2006 Biotransformation of fluticasone: in vitro characterization. Drug Metabolism and Disposition 34 1035-1040. (doi:10.1124/dmd.105.009043)

10 He B, Shi L, Qiu J, Tao L, Li R, Yang L \& Zhao S 2011 A functional polymorphism in the CYP3A4 gene is associated with increased risk of coronary heart disease in the Chinese Han population. Basic and Clinical Pharmacology and Toxicology 108 208-213. (doi:10.1111/ j.1742-7843.2010.00657.x)

Received in final form 23 February 2017 Accepted 2 March 2017 